PESQUIMAT, Revista de la F.C.M. de la

Universidad Nacional Mayor de San Marcos

Vol. XI - Nº1 Pgs. 1-20 Lima - Perú Oct. 2008

\title{
SOLUCIÓN LOCAL PARA UNA CLASE DE ECUACIONES NO-LINEALES ABSTRACTAS TIPO KIRCHHOFF- CARRIER
}

\author{
Raúl Moisés Izaguirre Maguiña ${ }^{1}$ \& Víctor Arturo Martínez León ${ }^{2}$ \\ Julio Flores Dionicio ${ }^{3}$
}

Resumen.- Sean, $(V, a(u, v)),(H,(u, v))$ espacios de Hilbert y la inmersión $V \subset H$ es densa y compacta. Consideramos el problema abstracto:

$$
\left\{B u^{\prime \prime}+M\left(|u|_{0}^{2}\right) A u \doteq f\right.
$$

Donde $B: H \rightarrow H$ es un operador lineal, simétrico y positivo, $\left(W,|u|_{0}\right)$, es un espacio de Banach, la función no lineal $M$ es de clase $C^{1}$ y estrictamente positiva. En el trabajo demostramos la existencia local y la unicidad de solución local del problema (*).

Palabras claves: Espacio de Hilbert, espacio de Banach, problema abstracto, ecuación de Kirchoff-Carrier, solución local, teorema del punto fijo de Rothe.

Abstract.- Let, $(V, a(u, v)),(H,(u, v))$ Hilbert spaces and the immersion $V \subset H$ is dense and compact. We consider the abstract problem:

$$
\left\{B u^{\prime \prime}+M\left(|u|_{0}^{2}\right) A u=f\right.
$$

Where $B: H \rightarrow H$ is a linear simetric and positive operator, $\left(W,|u|_{0}\right)$, is a Banach space, the non linear $M$ is $C^{1}$ class and strictively positive. In this article we prove the existence and unicity of the local solution of the problem (*).

Key words: Hilbert space, Banach space, abstract problem, Kirchoff-Carrier equation, local solution, fixed point theorem of Rothe.

\footnotetext{
${ }^{1}$ UNMSMS, Facultad de Ciencias Matemáticas, e-mail: raul_izaguirre2222@yahoo.es

${ }^{2}$ UNMSMS, Facultad de Ciencias Matemáticas, e-mail: vicaml19@gmail.com

${ }^{3}$ UNMSMS, Facultad de Ciencias Matemáticas, e-mail: juliofloresd@hotmail.com
} 


\section{DESCOMPOSICIÓN ESPECTRAL}

Sean, $(V, a(u, v)),(H,(u, v))$ espacios de Hilbert y la inmersión $V \subset H$ es densa y compacta.

Sea $b: H \times H \rightarrow \mathbb{R}$, una forma bilineal, simétrica (luego continua) y positiva. Esto es:

(1) $b(u, v)=b(v, u), u, v \in H$

(2) $b(u, u)>0, u \neq 0$

Entonces $(H, b(u, v))$ es un espacio pre Hilbert. Sea $(\widehat{H}, \widehat{b}(u, v))$ su completación que es un espacio de Hilbert. Observemos que $b(u, v)=\widehat{b}(i u, i v), \forall u, v \in H$, donde $i: H \rightarrow \widehat{H}$ es el operador de inmersión que es lineal, isométrico de rango denso.

Entonces $V \subset \widehat{H}$, con inmersión densa continua y compacta. Sea $S$ el operador lineal, auto adjunto, determinado por la terna $\{V, \widehat{H}, a(u, v)\}$.

Se tiene que: $S: D(S) \subset V \rightarrow \widehat{H}$ es biyectivo, con operador inverso $S^{-1}: \widehat{H} \rightarrow D(S)$ compacto, $D(S)$ es denso en $V$ y en $\widehat{H}$. Además se verifica la relación:

(3) $\widehat{b}(S u, i v)=a(u, v), u \in D(S), v \in V$.

Observación 1. El espacio $\widehat{H}$ es isomorfo al espacio dual $H^{\prime}$ de $(H, b(u, v))$ y que la inmersión $i: H \rightarrow H^{\prime}$, es identificada con $i=B: H \rightarrow H^{\prime}$.

Desde la teoría espectral para operadores autoadjuntos y compactos, existe una sucesión ortonormal $\left\{w_{k}\right\}$ de autovectores de $S^{-1}$, para los cuales la correspondiente sucesión de autovalores $\left\{\mu_{k}\right\}$ converge a cero y los autovectores forman una base para $R g\left(S^{-1}\right)=D(A)$. Entonces tenemos que existe una base numerable $\left(w_{k}\right)_{k \geq 1}$ de $V$ y una sucesión creciente de números positivos $\left(\lambda_{k}\right)_{k \geq 1}$ tales que:

(4) $\lambda_{j} b\left(w_{j}, v\right)=a\left(w_{j}, v\right), \forall v \in V ; j=1,2, \ldots$

(5) $b\left(w_{j}, w_{i}\right)=\delta_{i j} ; a\left(w_{j}, w_{j}\right)=\lambda_{j} ; j=1,2, \ldots$

(6) $S w_{j}=\lambda_{j} w_{j} ; j=1,2, \ldots$

Desde la teoría espectral para $\alpha \in \mathbb{R}$, podemos definir la potencia $S^{\alpha}$, con dominio $D\left(S^{\alpha}\right)=\left\{u ; \sum_{v=1}^{\infty} \lambda_{v}^{2 \alpha}\left|b\left(u, w_{v}\right)\right|^{2}<\infty\right\}$ 


$$
\begin{aligned}
& S^{\alpha} u=\sum_{v=1}^{\infty} \lambda_{v}^{\alpha} b\left(u, w_{v}\right) w_{v}, \forall u \in D\left(S^{\alpha}\right) \\
& (u, v)_{\alpha}=\sum_{v=1}^{\infty} \lambda_{v}^{2 \alpha} b\left(u, w_{v}\right) b\left(v, w_{v}\right) \\
& |u|_{\alpha}^{2}=\left|S^{\alpha} u\right|^{2}=\sum_{v=1}^{\infty} \lambda_{v}^{2 \alpha}\left|b\left(u, w_{v}\right)\right|^{2}
\end{aligned}
$$

se tiene que $\left(D\left(S^{\alpha}\right),(u, v)_{\alpha}\right)$ es un espacio de Hilbert y si $\alpha<\beta$ la inmersión de $D\left(S^{\beta}\right) \subset D\left(S^{\alpha}\right)$ es compacta $\forall \alpha, \beta \in \mathbb{R}$

PROBLEMA LINEAL. Sea $T>0$. Consideremos el problema de hallar solución para la ecuación:

(7) $\left\{\begin{aligned} B u^{\prime \prime}(t)+M(t) A u(t) & =B f(t) \\ u(0)=u_{0} ; u^{\prime}(0) & =u_{1}\end{aligned}\right.$ en $V^{\prime}$

Trabajando formalmente, sea $v \in V$. Entonces

$$
b\left(u^{\prime \prime}(t), v\right)+M(t) a(u(t), v)=b(f(t), v)
$$

Por (3)

$$
\widehat{b}\left(i u^{\prime \prime}, i v\right)+M(t) \widehat{b}(S u(t), i v)=\widehat{b}(i f(t), i v)
$$

Entonces desde que $V$ es denso en $\widehat{H}$, el sistema es equivalente con:

(8) $\left\{\begin{array}{c}u^{\prime \prime}(t)+M(t) S u(t)=f(t) \\ u(0)=u_{0} ; u^{\prime}(0)=u_{1}\end{array}\right.$ en $H^{\prime}$

Consideremos la función $\Psi$ tal que:

(9) $\Psi \in C^{1}([0, T]), \Psi(t) \geq m_{0}>0 \forall s \in[0, T]$

(10) $\Psi^{\prime} \in L^{\infty}(0, T) ;\left|\Psi^{\prime}\right|_{L^{\infty}(0, T)} \leq M_{1}$

Teorema 1. Sea $\alpha \in \mathbb{R}$ y $\Psi$ que verifica las condiciones (1), (2).

(11) $u_{0} \in D\left(S^{\alpha+1}\right)$

(12) $u_{1} \in D\left(S^{\alpha+1 / 2}\right)$

(13) $f \in L^{2}\left(0, T ; D\left(S^{\alpha+1 / 2}\right)\right)$

Entonces existe una única solución $u$ del problema (8) tal que: 
(14) $u \in L^{\infty}\left(0, T ; D\left(S^{\alpha+1}\right)\right)$

(15) $u^{\prime} \in L^{\infty}\left(0, T ; D\left(S^{\alpha+1 / 2}\right)\right)$

(16) $u^{\prime \prime} \in L^{2}\left(0, T ; D\left(S^{\alpha}\right)\right)$

(17) $u^{\prime \prime}+\Psi(t) S u=f$ en $L^{2}\left(0, T ; D\left(S^{\alpha}\right)\right)$

La solución satisface el siguiente estimado de energía:

(18) $E(t)=\left|S^{\alpha+1 / 2} u^{\prime}(t)\right|^{2}+\left|S^{\alpha+1} u(t)\right|^{2} \leq D_{0} \exp \left(D_{1} t\right)$

donde

$$
\begin{aligned}
& C_{0}=\left|S^{(2 \alpha+1) / 2} u_{1}\right|^{2}+\Psi(0)\left|S^{\alpha+1} u_{0}\right|^{2}+|f|_{L^{2}\left(0, T ; D\left(S^{(2 \alpha+1) / 2}\right)\right)}^{2} \\
& C_{1}=\min \left\{1, m_{0}\right\} ; C_{2}=\max \left\{1, M_{1}\right\} \\
& D_{0}=\frac{C_{0}}{C_{1}} ; D_{1}=\frac{C_{2}}{C_{1}}
\end{aligned}
$$

Demostración. Sea $V_{m}=\left[w_{1}, w_{2}, \ldots, w_{m}\right] \subset V$. Luego $V_{m}$ es un subespacio de $V$ de dimensión finita $m$, e invariante bajo la acción del operador $S^{k}$.

Sea entonces:

(19) $u_{m}(t)=\sum_{i=1}^{m} g_{i m}(t) w_{i} \in V_{m}$

Donde las funciones $g_{i m}$, son determinadas por la solución del siguiente sistema de ecuaciones diferenciales ordinarias lineales:

(20) $\left\{\begin{array}{l}b\left(u_{m}^{\prime \prime}(t), w_{j}\right)+M(t) a\left(u_{m}(t), w_{j}\right)=a\left(f(t), w_{j}\right) \quad \forall j=1,2, \ldots, m \\ u_{m}(0)=u_{0 m} ; u_{m}^{\prime}(0)=u_{1 m}\end{array}\right.$

Por la selección de la base especial, el sistema es equivalente con el sistema lineal de ecuaciones diferenciales ordinarias:

$$
\left\{\begin{array}{l}
g_{j m}^{\prime \prime}(t)+M(t) \sum_{k=1}^{m} \lambda_{k} g_{k m}(t)=b\left(f(t), w_{j}=f_{j}(t)\right) \\
g_{j m}(0)=b\left(u_{0}, w_{j}\right)=a_{j} \\
g_{j m}^{\prime}(0)=b\left(u_{1}, w_{j}\right)=b_{j} \\
j=1,2, \ldots, m
\end{array}\right.
$$

El sistema de ecuaciones diferenciales lineales $(* *)$, admite una única solución en un intervalo $\left[0, t_{m}\right\rangle$, de donde seguimos la existencia de las soluciones aproximadas $u_{m}$ para 
$m \geq 1$. Seguimamente debemos obtener estimado a priori para la sucesión $\left\{u_{m}\right\}$ de modo que podamos prolongarlas a un intervalo uniforme de existencia.

ESTIMADO A PRIORI 1. Por linealidad la igualdad en (20) se verifica para todo $v \in V_{m}$. Entonces haciendo $v=S^{2 \alpha+2} u_{m}^{\prime}(t)$, obtenemos:

(21) $\left(S^{\alpha+1 / 2} u_{m}^{\prime \prime}(t), S^{\alpha+1 / 2} u_{m}^{\prime}(t)\right)+M(t)\left(S^{\alpha+1} u_{m}(t), S^{\alpha+1} u_{m}^{\prime}(t)\right)=\left(S^{\alpha+1 / 2} f(t), S^{\alpha+1 / 2} u_{m}^{\prime}(t)\right)$

Luego:

$\frac{d}{d t}\left\{\left|S^{\alpha+1 / 2} u_{m}^{\prime}(t)\right|^{2}+M(t)\left|S^{\alpha+1} u_{m}(t)\right|\right\} \leq\left|S^{\alpha+1 / 2} f(t)\right|^{2}+\left|S^{\alpha+1 / 2} u_{m}^{\prime}(t)\right|^{2}+M_{1}\left|S^{\alpha+1} u_{m}(t)\right|^{2}$

Ahora integrando en esta desigualdad:

(22)

$$
\begin{aligned}
& \left|S^{\alpha+1 / 2} u_{m}^{\prime}(t)\right|^{2}+m_{0}\left|S^{\alpha+1} u_{m}(t)\right|^{2} \leq\left|S^{\alpha+1 / 2} u_{m}^{\prime}(t)\right|^{2}+\dot{M}(t)\left|S^{\alpha+1} u_{m}^{\prime}(t)\right|^{2} \leq \\
& \leq \int_{0}^{t}\left|S^{\alpha+1 / 2} f(s)\right|^{2} d s+\int_{0}^{t}\left|S^{\alpha+1 / 2} u_{m}^{\prime}(t)\right|^{2} d s+\left|S^{\alpha+1 / 2} u_{m}^{\prime}(0)\right|^{2}+M(0)\left|S^{\alpha+1} u_{m}(0)\right|^{2} \\
& +M_{1} \int_{0}^{t}\left|S^{\alpha+1} u_{m}(s)\right|^{2} d s \leq\left|u_{1}\right|_{\alpha+1 / 2}^{2}+M_{0}\left|u_{0}\right|_{\alpha+1}^{2}+T|f|_{L^{\infty}\left(0, T ; D\left(S^{\alpha+1 / 2}\right)\right)}^{2}+ \\
& +\int_{0}^{t}\left|S^{\alpha+1 / 2} u_{m}^{\prime}(s)\right|^{2} d s+M_{1} \int_{0}^{t}\left|S^{\alpha+1} u_{m}(s)\right|^{2} d s
\end{aligned}
$$

Entonces

(23) $\eta(t)=\left|S^{\alpha+1 / 2} u_{m}^{\prime}(t)\right|^{2}+\left|S^{\alpha+1} u_{m}(t)\right|^{2} \leq D_{0}+D_{1} \int_{0}^{t} \eta(s) d s$

Aplicando el lema de Gronwall obtenemos:

$$
\left|S^{\alpha+1 / 2} u_{m}^{\prime}(t)\right|^{2}+\left|S^{\alpha+1} u_{m}(t)\right|^{2} \leq D_{0} e^{D_{1} t} \leq D_{0} e^{D_{1} T}
$$

Luego

(25) $\left(u_{m}\right)$ es acotada en $L^{\infty}\left(0, T ; D\left(S^{\alpha+1}\right)\right)$

(26) $\left(u_{m}^{\prime}\right)$ es acotada en $L^{\infty}\left(0, T ; D\left(S^{\alpha+1 / 2}\right)\right)$ 
ESTIMADO A PRIORI 2. Multiplicando en la ecuación aproximada por $w_{j}$ y luego sumando, obtenemos:

(27) $\sum_{j=1}^{m}\left(u_{m}^{\prime \prime}(t), w_{j}\right) w_{j}+\sum_{j=1}^{m}\left(S u_{m}(t), w_{j}\right) w_{j}=\sum_{j=1}^{m}\left(f(t), w_{j}\right) w_{j}$

Por la definición de la proyección, $P_{m}: V \rightarrow V_{m}$ :

(28) $u_{m}^{\prime \prime}(t)=-M(t) S u_{m}(t)+P_{m} f(t)=-M(t) S u_{m}(t)+f_{m}(t)$

Luego:

(29) $\left|S^{\alpha} u_{m}^{\prime \prime}(t)\right| \leq M_{0}\left|S^{\alpha+1} u_{m}(t)\right|+d_{4}\left|S^{\alpha+1 / 2} f(t)\right|$

Por lo cual

(30) $\left(u_{m}^{\prime \prime}\right)$ es acotada en $L^{2}\left(0, T ; D\left(S^{\alpha}\right)\right)$.

\section{CONVERGENCIA DE LAS SOLUCIONES APROXIMADAS}

Para tratar la convergencia de las soluciones aproximadas utilizamos el siguiente:

Lema 2. Sean $X, Y, Z$ espacios de Banach tales que $X \subset Y \subseteq Z$, con inmersiones continuas y la inmersión $X \subset Y$ es compacta. Sea

$$
W=\left\{u \in L^{p}(0, T ; X) ; u^{\prime} \in L^{q}(0, T ; Z)\right\}
$$

donde $u^{\prime}$ denota la derivada generalizada de $u:[0, T] \rightarrow X$, sobre $(0, T)$. Entonces

(31) Si $p=\infty, q>1$ entonces $W \subseteq C([0, T] ; Z)$ es compacta.

(32) Si $1 \leq p<\infty, q=1$ entonces $W \subseteq L^{p}(0, T ; Y)$ es compacta.

Demostración. (Ver J. Simon [15]).

Por los estimados 1, 2, tenemos que:

(33) $\left(u_{m}\right)$ es acotada en $L^{\infty}\left(0, T ; D\left(S^{\alpha+1}\right)\right)$

(34) $\left(u_{m}^{\prime}\right)$ es acotada en $L^{\infty}\left(0, T ; D\left(S^{\alpha+1 / 2}\right)\right)$

(35) $\left(u_{m}^{\prime \prime}\right)$ es acotada en $L^{2}\left(0, T ; S^{\alpha}\right)$ 
Luego, existe una subsucesión de $\left(u_{m}\right)$ que continuamos denotando de la misma forma tal que:

(36) $u_{m} \rightarrow u$ débil-* en $L^{\infty}\left(0, T ; D\left(S^{\alpha+1}\right)\right)$

(37) $u_{m}^{\prime} \rightarrow u^{\prime}$ débil en $L^{2}\left(0, T ; D\left(S^{\alpha+1 / 2}\right)\right)$

(38) $u_{m}^{\prime \prime} \rightarrow u^{\prime \prime}$ débil en $L^{2}\left(0, T ; D\left(S^{\alpha}\right)\right)$

Por las inmersiones continuas y compactas: $D\left(A^{\alpha+1}\right) \subset D\left(A^{(2 \alpha+1) / 2}\right) \subset D\left(A^{\alpha}\right)$, el Lema 2, existe una adecuada subsucesión de $\left(u_{m}\right)$ que continuamos denotando de la misma forma, tal que:

(39) $u_{m} \stackrel{m \rightarrow \infty}{\longrightarrow} u$ fuerte en $C^{0}\left([0, T] ; D\left(S^{(2 \alpha+1) / 2}\right)\right) \subset C^{0}\left([0, T] ; W_{0}\right)$

(40) $u_{m}^{\prime} \stackrel{m \rightarrow \infty}{\longrightarrow} u^{\prime}$ fuerte en $C^{0}\left([0, T] ; D\left(S^{\alpha}\right)\right)$

De (37), (39) y procediendo de forma estándar obtenemos que la función $u$ es solución del problema (3) y verifica:

(41) $u^{\prime \prime}(t) \Psi(t) S u(t)=f(t)$ en $L^{2}\left(0, T ; D\left(S^{\alpha}\right)\right)$

Para demostrar que la solución $u$ verifica el estimado de energía (18), probaremos primero que:

(42) $u_{m}(t) \rightarrow u(t)$ débil en $D\left(S^{\alpha+1}\right) \forall t \in[0, T]$

(43) $u_{m}^{\prime}(t) \rightarrow u^{\prime}(t)$ débil en $D\left(S^{(2 \alpha+1) / 2}\right) \forall t \in[0, T]$

(44) $u_{m}^{\prime \prime}(t) \rightarrow u^{\prime \prime}(t)$ débil en $D\left(S^{\alpha}\right) \forall t \in[0, T]$

Sea $v \in V_{k} ; m \geq k$. Tenemos

(45) $\left(S^{\alpha+1} u_{m}(t), S^{\alpha+1} v\right)=\left(S^{\alpha+1 / 2} u_{m}(t), S^{\alpha+3 / 2} v\right) \rightarrow$

$\rightarrow\left(S^{\alpha+1 / 2} u(t), S^{\alpha+3 / 2} v\right)=\left(S^{\alpha+1} u(t), S^{\alpha+1} v\right)$

Observación: Por (40)

(46) $\left|\left(S^{\alpha+1 / 2} u_{m}(t)-S^{\alpha+1 / 2} u(t), S^{\alpha+3 / 2} v\right)\right| \leq\left|S^{\alpha+1 / 2} u_{m}(t)-S^{\alpha+1 / 2} u(t)\right|\left|S^{\alpha+3 / 2} v\right| \stackrel{m \rightarrow \infty}{\longrightarrow} 0$ 
(47) $\left(S^{\alpha+1 / 2} u_{m}^{\prime}(t), S^{\alpha+1 / 2} v\right)=\left(S^{\alpha} u_{m}^{\prime}(t), S^{\alpha+1} v\right) \rightarrow$

$\rightarrow\left(S^{\alpha} u^{\prime}(t), S^{\alpha+1 / 2} v\right)=\left(S^{\alpha+1 / 2} u^{\prime}(t), S^{\alpha+1 / 2} v\right)$

Asimismo

(48) $\left(S^{\alpha} u_{m}^{\prime \prime}(t), S^{\alpha} v_{k}\right)=-M(t)\left(S^{\alpha+1} u_{m}(t), S^{\alpha} v_{k}\right)+\left(S^{\alpha} f_{m}(t), S^{\alpha} v_{k}\right)=$

$$
\begin{aligned}
& =-M(t)\left(S^{\alpha+1} u_{m}(t), S^{\alpha} v_{k}\right)+\left(f_{m}(t), S^{2 \alpha} v_{k}\right) \\
& =-M(t)\left(S^{\alpha+1} u_{m}(t), S^{\alpha} v_{k}\right)+\left(f(t), S^{2 \alpha} v_{k}\right) \rightarrow-M(t)\left(S^{\alpha+1} u(t), S^{\alpha} v_{k}\right)+\left(S^{\alpha} f(t), S^{\alpha} v_{k}\right) \\
& =\left(S^{\alpha} u^{\prime \prime}, S^{\alpha} v_{k}\right)
\end{aligned}
$$

Luego por el Estimado 1 y (44), (45), obtenemos que:

(49) $\left|S^{\alpha+1 / 2} u^{\prime}(t)\right|^{2}+\left|S^{\alpha+1} u(t)\right|^{2} \leq \lim _{m \rightarrow \infty}\left\{\left|S^{\alpha+1 / 2} u_{m}^{\prime}(t)\right|^{2}+\left|S^{\alpha+1} u_{m}(t)\right|^{2}\right\} \leq D_{0} e^{D_{1} T}$

UNICIDAD. Sean $u, z$ dos soluciones de (41) y $y=u-z$. Entonces

(50) $y \in L^{\infty}\left(0, T ; D\left(S^{\alpha+1}\right)\right)$

(51) $y^{\prime} \in L^{\infty}\left(0, T ; D\left(S^{(2 \alpha+1) / 2}\right)\right)$

(52) $y^{\prime \prime} \in L^{2}\left(0, T ; D\left(S^{\alpha}\right)\right)$

(53) $y(0)=0 ; y^{\prime}(0)=0$

Y satisface la ecuación:

(54) $y^{\prime \prime}+\Psi(t) A y=0$ en $L^{2}\left(0, T ; D\left(S^{\alpha}\right)\right)$

Componiendo con $w=2 y^{\prime}(t) \in D\left(S^{\alpha}\right)$ en esta ecuación, obtenemos

(55) $\frac{d}{d t}\left\{\left|S^{\alpha} y^{\prime}(t)\right|^{2}+\Psi(t)\left|S^{\alpha+1 / 2} y(t)\right|^{2}\right\}=\Psi^{\prime}(t)\left|S^{\alpha+1 / 2} y(t)\right|^{2} \leq M_{1}\left|S^{\alpha+1 / 2} y(t)\right|^{2}$

integrando de 0 a $t$ y teniendo en cuenta que $y(0)=y^{\prime}(0)=0$

(56) $\eta(t)=\left|S^{\alpha} y^{\prime}(t)\right|^{2}+m_{0}\left|S^{(2 \alpha+1) / 2} y(t)\right|^{2} \leq C \int_{0}^{t} \eta(s) d s$

por el lema de Gronwall y desde que $y \in C^{0}\left([0, T] ; D\left(S^{\alpha+1 / 2}\right)\right), y^{\prime} \in C^{0}\left([0, T] ; D\left(S^{\alpha}\right)\right)$

(57) $S^{\alpha} y^{\prime}(t)=S^{\alpha+1 / 2} y(t)=0, \forall t \in[0, T]$. 
Luego $u=z$.

PROBLEMA NO-LINEAL En esta parte demostraremos la existencia de solución local del problema:

(58) $u^{\prime \prime}+M\left(|u(t)|_{0}^{2}\right) S u=f ;$ en $L^{2}\left(0, T_{0} ; D\left(S^{\alpha}\right)\right)$

(59) $u(0)=u_{0} ; u^{\prime}(0)=u_{1}$

donde:

(60) $\left(W_{0},|u|_{0}\right)$ es un espacio de Banach real, reflexivo, tal que su correspondiente espacio dual $W_{0}^{*}$ es estrictamente convexo y verifiva la condición:

(61) $D\left(S^{\alpha+1 / 2}\right) \subset W_{0}$.

Entonces de (60) y de la teoría de los operadores máximo monótonos, obtenemos las siguientes propiedades del operador de dualidad.

Teorema 2. Si $W$ es un espacio de Banach, real reflexivo tal que su espacio dual $W^{*}$, es estrictamente convexo. Entonces la aplicación de dualidad $J: W \rightarrow W^{*}$ es simple valuada, demicontinua, máximo monótona, acotada, coercitiva y

$$
|J u|_{W^{*}}=|u|_{W}
$$

La función $u \rightarrow|u|_{W}^{2}$ es $G$-diferenciable sobre $W$ y si $\Psi(u)=|u|_{W}^{2}$, entonces

$$
\Psi^{\prime}(u)=2 J u, \forall u \in W
$$

\section{HIPÓTESIS SOBRE LA FUNCIÓN M}

H-1 $M \in C^{1}\left(\mathbb{R}, \mathbb{R}^{+}\right)$

$\mathrm{H}-2 M(s)>m_{0}, \forall s \in \mathbb{R} ; \mathrm{y}$

H-3 $M, M^{\prime}$, son crecientes. 
DEFINICIÓN DE CONSTANTES. En esta parte definimos diversas constantes que aparecen en esta parte del trabajo.

\section{Constantes de inmersión:}

C-1 $|v|_{0} \leq d_{0}\left|S^{\alpha+1} v\right|, \forall v \in D\left(S^{\alpha+1}\right)$

$\mathrm{C}-2|v|_{0} \leq d_{1}\left|S^{\alpha+3 / 2} v\right|, \forall v \in D\left(S^{\alpha+3 / 2}\right)$

C-3 $\left|S^{\alpha+1 / 2} v\right| \leq b_{1}\left|S^{\alpha+1} v\right|, \forall v \in D\left(S^{\alpha+1}\right)$

C-4 $\left|S^{\alpha+1} v\right| \leq b_{2}\left|S^{\alpha+3 / 2} v\right|, \forall v \in D\left(S^{\alpha+3 / 2}\right)$

Consideremos los siguientes espacios de Banach:

$W=\left\{v \in L^{\infty}\left(0, T ; D\left(S^{\alpha+1}\right)\right) ; v^{\prime} \in L^{\infty}\left(0, T ; D\left(S^{\alpha+1 / 2}\right)\right)\right\}$.

$Z=\left\{v \in L^{\infty}\left(0, T ; D\left(S^{\alpha+3 / 2}\right)\right) ; v^{\prime} \in L^{\infty}\left(0, T ; D\left(S^{\alpha+1}\right)\right) ; v^{\prime \prime} \in L^{2}\left(0, T ; D\left(S^{\alpha+1 / 2}\right)\right)\right\}$

Entonces $Z \subset W$ con inmersión continua y compacta. En efecto, sea $\left(v_{m}\right)$ una sucesión acotada en $Z$, entonces

$\left(v_{m}\right)$ es acotada en $L^{\infty}\left(0, T ; D\left(S^{\alpha+3 / 2}\right)\right)$

$\left(v_{m}^{\prime}\right)$ es acotada en $L^{\infty}\left(0, T ; D\left(S^{\alpha+1}\right)\right)$

$\left(v_{m}^{\prime \prime}\right)$ es acotada en $L^{2}\left(0, T ; D\left(S^{\alpha+1 / 2}\right)\right)$

Desde el Teorema de Simón, podemos obtener una subsucesión $\left(v_{k}\right) \subset\left(v_{m}\right)$ y una función $v$, tal que:

$(62) v_{k} \rightarrow v$, fuerte en $C^{0}\left([0, T] ; D\left(S^{\alpha+1}\right)\right) \subset L^{\infty}\left(0, T ; D\left(S^{\alpha+1}\right)\right)$

(63) $v_{k}^{\prime} \rightarrow v^{\prime}$, fuerte en $C^{0}\left([0, T] ; D\left(S^{\alpha+1 / 2}\right)\right) \subset L^{\infty}\left(0, T ; D\left(S^{\alpha+1 / 2}\right)\right)$

Luego $v_{k} \rightarrow v$, en $W$.

Teorema 2. Sean $v \in W$,

(64) $u_{0} \in D\left(S^{\alpha+3 / 2}\right)$

(65) $u_{1} \in D\left(S^{\alpha+1}\right)$

(66) $f \in L^{\infty}\left(0, T ; D\left(S^{\alpha+1}\right)\right)$

Entonces, existe una única solución $u \in Z$ del problema: 
(67) $u^{\prime \prime}+M\left(|v(t)|_{0}^{2}\right) S u=f$ en $L^{2}\left(0, T ; D\left(S^{\alpha+1 / 2}\right)\right)$

(68) $u(0)=u_{0} ; u^{\prime}(0)=u_{1}$

Demostración: Procediendo como en la demostración del Teorema 1, haciendo $\Psi(t)=M\left(|v(t)|_{W}\right)$, reemplazando $\alpha$ por $\alpha+1 / 2$ en el Lema 1 , se obtiene que para todo $v \in W$, existe una única función $u$, tal que:

(69) $u \in L^{\infty}\left(0, T_{0} ; D\left(S^{\alpha+3 / 2}\right)\right)$

(70) $u^{\prime} \in L^{\infty}\left(0, T_{0} ; D\left(S^{\alpha+1}\right)\right)$

(71) $u^{\prime \prime} \in L^{2}\left(0, T_{0} ; D\left(S^{\alpha+1 / 2}\right)\right)$

(72) $u^{\prime \prime}+\Psi(t) S u=f$ en $L^{2}\left(0, T ; D\left(\dot{S}^{\alpha+1 / 2}\right)\right)$

(73) $u(0)=u_{0}, u^{\prime}(0)=u_{1}$

y además satiface el estimado de energía:

(74) $\left|S^{\alpha+1} u^{\prime}(t)\right|^{2}+\left|S^{\alpha+3 / 2} u(t)\right|^{2} \leq D_{0} e^{D_{1} T}$

Luego $u \in Z$.

Consideremos

$B_{K}=\left\{v \in W ;|v|_{W}^{2}=\left|S^{\alpha+1 / 2} v^{\prime}(t)\right|^{2}+\left|S^{\alpha+1} v(t)\right|^{2} \leq K^{2}\right\}$

La bola en $W$ con centro en 0 y radio $K$.

Entonces por la hipótesis (H-3) y (74), se tiene que para $v \in B_{K}$ :

(75) $|v(t)|_{0} \leq d_{0} K,\left|v^{\prime}(t)\right|_{0} \leq d_{1} K$

(76) $M\left(|v(t)|_{0}^{2}\right) \leq M\left(d_{0}^{2} K^{2}\right)=M_{0}$

(77) $M^{\prime}\left(|v(t)|_{0}^{2}\right)|v(t)|_{0}\left|v^{\prime}(t)\right|_{0} \leq d_{0} d_{1} ; \quad M^{\prime}\left(d_{0}^{2} K^{2}\right) K^{2}=M_{1}=M_{1}(K)$

Supongamos que los datos iniciales son suficientemente pequeños tales que

(78) $\max \left\{b_{1}^{2}, b_{2}^{2}\right\} D_{0} e^{D_{1} T} \leq K^{2}$. 
Teorema 3. Sean $u_{0}, u_{1}, f$, datos iniciales que satisfacen las condiciones del Teorema 2 y la condición (78). Entonces existe una única solución $u \in B$ del problema (58), (59).

Demostración. Definimos la función $G: B_{K} \subset W \rightarrow B_{0} \subset Z$ tal que para $v \in B$, $G v=u \in Z$, es la única solución del problema:

(79) $u^{\prime \prime}+M\left(|v(t)|_{0}^{2}\right) S u=f$ en $L^{\infty}\left(0, T ; D\left(S^{\alpha+1 / 2}\right)\right)$

(80) $u(0)=u_{0} ; u^{\prime}(0)=u_{1}$

Donde:

$B_{0}=\left\{u \in Z ;\left|S^{\alpha+1} u^{\prime}(t)\right|^{2}+\left|S^{\alpha+3 / 2} u(t)\right|^{2} \leq D_{0} e^{D_{1} T},\left|S^{\alpha+1 / 2} u^{\prime \prime}(t)\right| \leq C_{2}\right\}$

Entonces para $u \in B_{0} \subset Z$, se tiene que:

$$
\begin{aligned}
\left|S^{\alpha+1 / 2} u^{\prime}(t)\right|^{2}+\left|S^{\alpha+1} u(t)\right|^{2} & \leq b_{1}^{2}\left|S^{\alpha+1} u^{\prime}(t)\right|^{2}+b_{2}^{2}\left|S^{\alpha+3 / 2} u(t)\right|^{2} \\
& \leq \max \left\{b_{1}^{2}, b_{2}^{2}\right\}\left(\left|S^{\alpha+1} u^{\prime}(t)\right|^{2}+\left|S^{\alpha+3 / 2} u(t)\right|^{2}\right) \\
& \leq \max \left\{b_{1}^{2}, b_{2}^{2}\right\} D_{0} e^{D_{1} T} \leq K^{2}
\end{aligned}
$$

Luego $B_{0} \subset B_{K}$. Sea $i: Z \rightarrow W$, la función de inmersión de $Z$ en $W$, que es lineal, continua y compacta. Desde que $B_{0}$ es un conjunto acotado en $Z, \overline{i\left(B_{0}\right)}$, es un conjunto compacto en $W$. Además $\overline{i\left(B_{0}\right)} \subset B_{K} \subset W$. Entonces $i G: B \subset W \rightarrow \overline{i\left(B_{0}\right)} \subset B_{K} \subset W$, aplica la bola cerrada de $W$ en un conjunto compacto de $W$ y $i G(\partial B) \subset B_{K}$.

Ahora demostraremos que la aplicación es continua.

Sean $\left(v_{m}\right) \subset W, v \in W$, tales que $v_{m} \rightarrow v$, en $W$. Entonces:

(81) $v_{m} \rightarrow v$, en $L^{\infty}\left(0, T ; D\left(S^{\alpha+1}\right)\right)$

$(82) v_{m}^{\prime} \rightarrow v^{\prime}$, en $L^{\infty}\left(0, T ; D\left(S^{\alpha+1 / 2}\right)\right)$

Sean $\left(u_{m}\right) \subset Z, u \in Z$, las correspondientes soluciones de los problemas:

(83) $u_{m}^{\prime \prime}+M\left(\left|v_{m}(t)\right|_{0}^{2}\right) A u_{m}=f$ en $L^{2}\left(0, T ; D\left(S^{\alpha+1 / 2}\right)\right)$

(84) $u_{m}(0)=u_{0} ; u_{m}^{\prime}(0)=u_{1}$

(85) $u^{\prime \prime}+M\left(|v(t)|_{0}^{2}\right) A u=f$ en $L^{2}\left(0, T ; D\left(S^{\alpha+1 / 2}\right)\right)$

(86) $u(0)=u_{0} ; u^{\prime}(0)=u_{1}$ 
Consideremos la sucesión $\left(z_{m}\right)=\left(u_{m}-u\right) \subset Z$. Entonces desde (83), (84), tenemos que

$(87) z^{\prime \prime}+M\left(\left|v_{m}(t)\right|_{0}^{2}\right) A z_{m}=\left(M\left(\left|v_{m}(t)\right|_{0}^{2}\right)-M\left(\left|v_{m}(t)\right|_{0}^{2}\right)\right) S u$ en $L^{2}\left(0, T ; D\left(S^{\alpha+1 / 2}\right)\right)$

\section{Entonces,}

(88) $z_{m}(0)=z_{m}^{\prime}(0)=0$

componiendo con $w=S^{\alpha+1 / 2} z_{m}(t)$, obtenemos:

(89) $\frac{d}{d t}\left\{\left|S^{\alpha+1} z_{m}^{\prime}(t)\right|^{2}+M\left(\left|v_{m}(t)\right|_{0}^{2}\right)\left|S^{\alpha+3 / 2} z_{m}(t)\right|^{2}\right\}$

$$
\begin{aligned}
& =2\left(M\left(|v(t)|_{0}^{2}\right)-M\left(\left|v_{m}(t)\right|_{0}^{2}\right)\right)\left(S^{\alpha+3 / 2} u(t), S^{\alpha+1 / 2} z_{m}(t)\right) \\
& +M^{\prime}\left(\left|v_{m}(t)\right|_{0}^{2}\right)\left\langle J v_{m}(t), v_{m}^{\prime}(t)\right\rangle\left|S^{\alpha+3 / 2} z_{m}(t)\right|^{2} \\
& \leq\left|M\left(|v(t)|_{0}^{2}\right)-M\left(\left|v_{m}(t)\right|_{0}^{2}\right)\right|^{2}\left|S^{\alpha+3 / 2} u(t)\right|^{2}+\left|S^{\alpha+1} z_{m}^{\prime}(t)\right|^{2}+M_{1}\left|S^{\alpha+3 / 2} z_{m}(t)\right|^{2} \\
& \leq C_{m}(t)+\left|S^{\alpha+1} z_{m}^{\prime}(t)\right|^{2}+M_{1}\left|S^{\alpha+3 / 2} z_{m}(t)\right|^{2}
\end{aligned}
$$

Donde:

(90) $C_{m}(t)=\left|M\left(\left|v_{m}(t)\right|_{0}^{2}\right)-M\left(|v(t)|_{0}^{2}\right)\right|\left|S^{\alpha+3 / 2} u(t)\right|^{2}$

Tenemos que:

$W \subset C^{0}\left([0, T], D\left(S^{\alpha+1 / 2}\right)\right) \subset C^{0}\left([0, T], W_{0}\right)$.

Luego $v_{m} \rightarrow v$, en $C^{0}\left([0, T], W_{0}\right)$, lo que implica que, $\left|v_{m}(t)\right|_{0}^{2} \rightarrow|v(t)|_{0}^{2}$ uniformemente y por la continuidad de $M, C_{m}(t) \rightarrow 0, m \rightarrow \infty$.

Entonces:

(91) $\left|S^{\alpha+1} z_{m}^{\prime}(t)\right|^{2}+\left|S^{\alpha+3 / 2} z_{m}(t)\right|^{2} \leq C_{m}(t) e^{C_{4} T}$

Tomando límite $m \rightarrow \infty$ y desde que $C_{m}(t) \rightarrow 0$, obtenemos que:

(92) $z_{m} \rightarrow z$, en $W$

Lo que demuestra que la aplicación $i G$ es continua. Para terminar la demostración, aplicaremos el siguiente resultado:

Teorema 4. (Rothe's). Sea $B$, denota una bola cerrada de un espacio vectorial normado $X$. Si $f$ aplica $B$ continuamente sobre un subconjunto compacto de $X$ y si $f(\partial B) \subset B$, entonces $f$ posee un punto fijo. 
Demostración. (Ver E. Zeidler [17]).

Por aplicación directa del Teorema de Rothe's, existe un punto fijo $u=i G u \in B_{k} \subset W$, solución del problema :

(93) $u^{\prime \prime}+M\left(|u(t)|_{0}^{2}\right) S u=f$ en $L^{2}\left(0, T: D\left(S^{\alpha}\right)\right)$

(94) $u(0)=u_{0} ; u^{\prime}(0)=u_{1}$

\section{UNICIDAD.}

Sean $u, z$ dos soluciones del problema (93) y (94) y $y=u-z$. Entonces se verifica que:

(95) $y \in L^{\infty}\left(0, T ; D\left(S^{\alpha+1}\right)\right)$

(96) $y^{\prime} \in L^{\infty}\left(0, T_{0} ; D\left(S^{\alpha+1 / 2}\right)\right)$

(97) $y^{\prime \prime} \in L^{2}\left(0, T_{0} ; D\left(S^{\alpha}\right)\right)$

(98) $y(0)=y^{\prime}(0)=0$

y satisface la ecuación :

(99) $y^{\prime \prime}+M\left(|u(t)|_{0}^{2}\right) S y=\left(M\left(|z(t)|_{0}^{2}\right)-M\left(|u(t)|_{0}^{2}\right)\right) S z$ en $L^{2}\left(0, T ; D\left(S^{\alpha+1 / 2}\right)\right)$

Componiendo con $w(t)=2 y^{\prime}(t)$ en esta ecuación y teniendo en cuenta que:

(100) $\left|M\left(|z(t)|_{0}^{2}\right)-M\left(|u(t)|_{0}^{2}\right)\right| \leq C|y(t)|_{0}$

obtenemos:

$$
\text { (101) } \begin{aligned}
& \frac{d}{d t}\left\{\left|S^{\alpha} y^{\prime}(t)\right|^{2}+\Psi(t)\left|S^{\alpha+1 / 2} y(t)\right|^{2}\right\} \\
& \leq C_{0}|y(t)|_{0}\left|S^{\alpha+1 / 2} z(t)\right|\left|S^{\alpha} y^{\prime}(t)\right|+\Psi^{\prime}(t)\left|S^{\alpha+1 / 2} y(t)\right|^{2} \\
& \leq C_{2}\left|S^{\alpha+1} y(t)\right|\left|S^{\alpha} y^{\prime}(t)\right|+C_{4}\left|S^{\alpha+1 / 2} y(t)\right|^{2} \\
& \leq C\left|S^{\alpha} y^{\prime}(t)\right|^{2}+C\left|S^{\alpha+1 / 2} y(t)\right|^{2}
\end{aligned}
$$

Donde $C$ representa diversas constantes que no dependen de $t$. Integrando de 0 a $t$ :

(102) $\eta(t)=\left|S^{\alpha} y^{\prime}(t)\right|^{2}+\left|S^{\alpha+1 / 2} y(t)\right|^{2} \leq C \int_{0}^{t} \eta(s) d s$ 
por el lema de Gronwall

$\left|S^{\alpha} y^{\prime}(t)\right|^{2}+\left|S^{\alpha+1 / 2} y(t)\right|^{2}=0$

Desde $y \in C^{0}\left([0, T] ; D\left(S^{\alpha+1 / 2}\right)\right), y^{\prime} \in C^{0}\left([0, T] ; D\left(S^{\alpha}\right)\right)$, obtenemos $S^{\alpha+1} y^{\prime}(t)=S^{\alpha+3 / 2} y(t)=0, \forall t \in[0, T]$.

Luego $u=z$.

\section{SOLUCIÓN PARA DATOS MÁS GENERALES.}

Sea el espacio de Hilbert:

(103) $X=\left\{f \in L^{2}\left(0, T ; D\left(S^{\alpha+1}\right)\right) ; f^{\prime} \in L^{2}\left(0, T ; D\left(S^{\alpha+1 / 2}\right)\right)\right\}$

(104) $Y=L^{2}\left(0, T ; D\left(S^{\alpha+1 / 2}\right)\right)$

Desde las inmersiones densas y compactas

$D\left(S^{\alpha+3 / 2}\right) \subset D\left(S^{\alpha+1}\right) \subset D\left(S^{\alpha+1 / " \prime}\right)$,

y el Lema 2, tenemos que:

$D\left(S^{\alpha+3 / 2}\right) \times D\left(S^{\alpha+1}\right) \times X \subset D\left(S^{\alpha+1}\right) \times D\left(S^{\alpha+1 / 2}\right) \times Y$ con inmersión compacta.

Sea $F$ la bola cerrada con centro en 0 y de radio $D_{0}^{1 / 2}$ en $D\left(S^{\alpha+3 / 2}\right) \times D\left(S^{\alpha+1}\right) \times X$ donde $D_{0}$ satisface la condición (76). Entonces $\bar{F}$ es compacta en $D\left(S^{\alpha+1}\right) \times D\left(S^{\alpha+1 / 2}\right) \times Y$ y $F$ es denso en $\bar{F}$.

TEOREMA 3. Sea $\left(u_{0}, u_{1}, f\right) \in \bar{F}$ : Entonces existe una única $u$ en la clase

(105) $u \in L^{\infty}\left(0, T ; D\left(S^{\alpha+1}\right)\right)$

(106) $u^{\prime} \in L^{\infty}\left(0, T ; D\left(S^{\alpha+1 / 2}\right)\right)$

(107) $u^{\prime \prime} \in L^{2}\left(0, T ; D\left(S^{\alpha}\right)\right)$

Solución del problema :

(108) $u^{\prime \prime}+M\left(|u(t)|_{0}^{2}\right) S u=f$ en $L^{2}\left(0, T ; D\left(S^{\alpha+1 / 2}\right)\right)$

(109) $u(0)=u_{0} ; u^{\prime}(0)=u_{1}$

Demostración. Desde que $\left(u_{0}, u_{1}, f\right) \in \bar{F}$, existe una sucesión $\left(u_{0 k}, u_{1 k}, f_{k}\right) \subset F$ tal que:

(110) $u_{0 k} \rightarrow u_{0}$, en $D\left(S^{\alpha+1}\right)$ 
(111) $u_{1 k} \rightarrow u_{1}$, en $D\left(S^{\alpha+1 / 2}\right)$

(112) $f_{k} \rightarrow f$, en $L^{2}\left(0, T ; D\left(S^{\alpha+1 / 2}\right)\right)$

Sea $\left(u_{k}\right) \subset Z$, las correspondientes soluciones dadas por el Teorema (3) de:

$(113) u_{k}^{\prime \prime}+M\left(\left|u_{k}(t)\right|_{0}^{2}\right) S u_{k}=f_{k}$ en $L^{2}\left(0, T ; D\left(S^{\alpha+1 / 2}\right)\right)$

(114) $u_{k}(0)=u_{0 k} ; u_{k}^{\prime}(0)=u_{1 k}$

La sucesión $\left(u_{k}\right)$ satisface:

(115) $\left|S^{\alpha+1 / 2} u_{k}^{\prime}(t)\right|^{2}+\left|S^{\alpha+1} u_{k}(t)\right|^{2} \leq K^{2}$

(116) $S^{\alpha} u_{k}^{\prime \prime}(t)=-M\left(\left|u_{k}(t)\right|_{0}^{2}\right) S^{\alpha+1} u_{k}(t)+S^{\alpha} f_{k}(t)$

Luego :

$\left(u_{k}\right)$ es acotada en $L^{\infty}\left(0, T: D\left(S^{\alpha+1}\right)\right)$

$\left(u_{k}^{\prime}\right)$ es acotada en $L^{\infty}\left(0, T: D\left(S^{\alpha+1 / 2}\right)\right)$

$\left(u_{k}^{\prime \prime}\right)$ es acotada en $L^{\infty}\left(0, T: D\left(S^{\alpha}\right)\right)$

Como en la demostración del Teorema 1 , podemos obtener una subsucesión $\left(u_{m}\right) \subset\left(u_{k}\right)$ y una función $u$, tal que:

(117) $u_{m} \rightarrow u$, débil $*$ en $L^{\infty}\left(0, T ; D\left(S^{\alpha+1}\right)\right)$

(118) $u_{m}^{\prime} \rightarrow u^{\prime}$, débil $*$ en $L^{\infty}\left(0, T ; D\left(S^{\alpha+1 / 2}\right)\right)$

(119) $u_{m}^{\prime \prime} \rightarrow u^{\prime \prime}$, débil $*$ en $L^{\infty}\left(0, T ; D\left(S^{\alpha}\right)\right)$

(120) $u_{m} \rightarrow u$, fuerte en $C^{0}\left([0, T] ; D\left(S^{\alpha+1 / 2}\right)\right) \subset C^{0}\left([0, T] ; W_{0}\right)$

(121) $u_{m}^{\prime} \rightarrow u^{\prime}$, fuerte en $C^{0}\left([0, T] ; D\left(S^{\alpha}\right)\right)$

Con estos estimados la función $u$, satisface la ecuación:

(122) $u^{\prime \prime}+M\left(|u(t)|_{0}^{2}\right) S u=f$ en $L^{2}\left(0, T ; D\left(S^{\alpha}\right)\right)$

(123) $u(0)=u_{0} ; u^{\prime}(0)=u_{1}$

En efecto, por (120), (121)

$(124) u_{m}(0)=u_{0 m} \rightarrow u_{0}=u(0)$ en $D\left(S^{\alpha+1}\right) \subset D\left(S^{\alpha+1 / 2}\right)$ 
(125) $u_{m}^{\prime}(0)=u_{1 m} \rightarrow u_{1}=u^{\prime}(0)$ en $D\left(S^{\alpha+1 / 2}\right) \subset D\left(S^{\alpha}\right)$

Ahora demostraremos la convergencia:

(126) $M\left(\left|u_{m}(t)\right|_{0}^{2}\right) S u_{m} \stackrel{m \rightarrow \infty}{\longrightarrow} M\left(|u(t)|_{0}^{2}\right) S u$ converge débil en $L^{\infty}\left(0, T_{0} ; D\left(S^{\alpha}\right)\right)$

Por (113), $\left|u_{m}(t)\right|_{0}^{2} \rightarrow|u(t)|_{0}^{2}$ en $C^{0}([0, T])$. Luego por la continuidad uniforme de $M$

(127) $M\left(\left|u_{m}(t)\right|_{0}^{2}\right) \rightarrow M\left(|u(t)|_{0}^{2}\right)$ en $C([0, T])$

Por otro lado, haciendo

(128) $N_{m}(t)=M\left(\left|u_{m}(t)\right|_{0}^{2}\right) ; N(t)=M\left(|u(t)|_{0}^{2}\right)$

(129) $\left|\int_{0}^{T}\left(N_{m}(t) S u_{m}(t)-N(t) S u(t), v(t)\right)_{\alpha}\right| d t \leq$

$\leq\left|\int_{0}^{T}\left(N_{p}(t) S u_{m}(t)-N(t) S u_{m}(t), v(t)\right)_{\alpha}\right| d t$

$\leq\left|\int_{0}^{T}\left(N(t) S z_{m}(t)-N(t) S u(t), v(t)\right)_{\alpha}\right| d t$

desde que la función $N \in C([0, T])$ y es acotada, $N v \in L^{1}\left(0, T ; D\left(S^{\alpha}\right)\right)$. Luego por (127), la segunda integral converge a 0. La primera integral es

$$
\begin{aligned}
& \left|\int_{0}^{T}\left(N_{m}(t) S u_{m}(t)-N(t) S u_{m}(t), v(t)\right)_{\alpha}\right| d t \leq \int_{0}^{T}\left|N_{p}(t)-N(t)\right|\left|S^{\alpha+1} u_{m}(t)\right|\left|S^{\alpha} u(t)\right| d t \\
& \leq \sup _{t \in[0, T]}\left|N_{p}(t)-N(t)\right|\left|S^{\alpha+1} u_{m}(t)\right| \int_{0}^{T}\left|S^{\alpha}(t)\right| d t \leq C \sup _{t \in[0, T]}\left|N_{m}(t)-N(t)\right| \stackrel{p \rightarrow \infty}{\longrightarrow} 0
\end{aligned}
$$

lo que demuestra (126). Luego :

(131) $u^{\prime \prime}+M\left(|u(t)|_{0}^{2}\right) S u=f$ en $L^{2}\left(0, T ; D\left(S^{\alpha}\right)\right)$

(132) $u(0)=u_{0}, u^{\prime}(0)=u_{1}$

\section{APLICACIONES}

1. Sea $\Omega \subset \mathbb{R}^{n}$ un abierto regular. Consideremos la función real $\rho: \Omega \rightarrow \mathbb{R}^{+}$, tal que $0<\rho(x) \leq \rho_{1}$ y $\rho^{-1} \in L^{\infty}(\Omega)$.

$V=H_{0}^{1}(\Omega), W=L^{p}(\Omega), H=L^{2}(\Omega)$

$1<\rho \leq \frac{2 n}{n-2}$, si $n \geq 3$, y $1<p<\infty$, si $n=1,2$ 
$M(s, r)=a+b s^{q / 2}, q \geq 2, a>0, b>0$

$A u=-\Delta u, B u=\rho u, S=\rho^{-1}(-\Delta), D(S)=D(-\Delta)=\left\{u \in H_{0}^{1}(\Omega) ; \Delta u \in L^{2}(\Omega)\right\}$.

$\left(u_{0}, u_{1}, f\right) \in D\left(S^{\alpha+1}\right) \times D\left(S^{\alpha+1 / 2}\right) \times X$, datos iniciales que satisfacen las condiciones del Teorema 3.

1.1 Sea $\alpha=0$, entonces $D\left(S^{1 / 2}\right)=H_{0}^{1}(\Omega) \subset L^{p}(\Omega)$. Haciendo $W_{0}=H_{0}^{1}(\Omega)$.

Entonces existe una única solución para los problemas:

$$
\begin{aligned}
& (133) \begin{cases}\rho(x) \frac{\partial^{2} u}{\partial t^{2}}+\left(a+b|\nabla u|_{L^{2}(\Omega)}^{q}\right) & \text { en } Q \\
u=0 & \text { en } \Sigma \\
u(0)=u_{0} ; u^{\prime}(0)=u_{1} & \text { en } \Omega\end{cases} \\
& \text { (134) } \begin{cases}\rho(x) \frac{\partial^{2} u}{\partial t^{2}}+\left(a+b|u|_{L^{p}(\Omega)}^{q}\right)(-\Delta u)=f & \text { en } Q . \\
u=0 & \text { en } \Sigma \\
u(0)=u_{0} ; u^{\prime}(0)=u_{1} & \text { en } \Omega\end{cases}
\end{aligned}
$$

En particular si $q=p=2$, (133) es conocida como la ecuación de Kirchhoff y (134) como la ecuación de Carrier.

$$
\begin{cases}\rho(x) \frac{\partial^{2} u}{\partial t^{2}}+\left(a+b \int_{\Omega}|u|^{p} d x\right)(-\Delta u)=f & \text { en } Q \\ u=0 & \text { en } \Sigma \\ u(0)=u_{0} ; u^{\prime}(0)=u_{1} & \text { en } \Omega\end{cases}
$$

1.2 Si $\alpha=-1 / 2$, entonces $D\left(S^{\alpha+1 / 2}\right)=D\left(S^{0}\right)=L^{2}(\Omega)$. En este caso podemos tratar con datos más generales y obtener soluciones para el problema de Carrier. En efecto si $u_{0} \in H_{0}^{1}(\Omega), u_{1} \in L^{2}(\Omega), f \in L^{2}(Q)$, entonces la solución $u$ del problema

$$
\begin{cases}\rho(x) \frac{\partial^{2} u}{\partial t^{2}}+\left(a+b \int_{\Omega}|u|^{2} d x\right)(-\Delta u)=f & \text { en } Q \\ u=0 & \text { en } \Sigma \\ u(0)=u_{0} ; u^{\prime}(0)=u_{1} & \text { en } \Omega\end{cases}
$$

Esta en la clase :

$$
\begin{aligned}
& -u \in L^{\infty}\left(0, T ; H_{0}^{1}(\Omega)\right) \\
& -u^{\prime} \in L^{\infty}\left(0, T ; L^{2}(\Omega)\right) \\
& -u^{\prime \prime} \in L^{2}\left(0, T ; H^{-1}(\Omega)\right)
\end{aligned}
$$


2. Sea $J: W \rightarrow W^{*}$ el operador de dualidad sobre $W$ y $\varphi(u)=|u|_{W}^{2}$. En el caso que $W$ sea un espacio de Hilbert $\varphi^{\prime}(u)=2 J u$, donde $J$ es el operador de Ríesz, $\langle J u, v\rangle_{W^{*} \times W}=(u, v)_{W} ; \forall u, v \in W$.

Como casos particulares de la aplicación 2, para $W=V=D\left(A^{1 / 2}\right)$, tenemos el problema abstracto de Kirchhoff :

$$
\left\{\begin{array}{l}
u^{\prime \prime}+M\left(\left|A^{1 / 2} u\right|^{2}\right) A u=f \\
u(0)=u_{0} ; u^{\prime}(0)=u_{1}
\end{array}\right.
$$

formulado por Lions J.L. en [9]. Si $W=H$, se tiene el problema de Carrier abstracto:

$$
\left\{\begin{array}{l}
u^{\prime \prime}+N\left(|u|_{H}^{2}\right) A u=f \\
u(0)=u_{0} ; u^{\prime}(0)=u_{1}
\end{array}\right.
$$

Una adecuada generalización de las aplicaciones (2), (3) es considerar $W=D\left(A^{\alpha}\right)$ para obtener:

$$
\left\{\begin{array}{l}
u^{\prime \prime}+N\left(\left|A^{\alpha} u\right|^{2}\right) A u=f \\
u(0)=u_{0} ; u^{\prime}(0)=u_{1}
\end{array}\right.
$$

conocido como el modelo abstracto de Kirchhoff-Carrier. 


\section{BIBLIOGRAFIA}

[1] CARRIER G.. F. On the non-linear vibration problern of the elastic string. Quart. Appl. Math. -3- (1945).

[2] COUSIN A., FROTA C., LARKIN N., MEDEIROS L. A. On the abstrac model of Kirchhoff Carrier Equation. Conim. In App. Analysis. - 3 - (1997).

[3] EBIHARA Y. MEDEIROS L.A. -MILLA. M. Local Solutions for a nonlinear degenerate hyperbolic equations. Nonlinear Analysis. Vol. 10 (1986).

[4] IZAGUIRRE R., VÉLIZ Y.Solución local para una clase de ecuaciones no-lineales degeneradas tipo Kirchhoff - Carrier 1 Seminario Internacional de Ecuaciones Diferenciales y Aplicaciones. Universidad Ricardo Palma - Lima - Perú -(1999).

[5] IZAGUIRRE R., VÉLIZ V. Solución Local para una clase de ecuaciones no-lineales de tipo Kirchhoff. Actas del 450 Seminario Brasileiro de Analise (1997).

[6] KIRCHHOFF G Vorlesungenuber mechanik. Teubner, Leipzig (1883).

[7] LIONS J.L. Quelques Methodes de Resolution des Probleme aux limites nonlinear Dunod. Paris. (1969).

[8] MEDEIROS LA., MILLA M. Solutionsfor the Equation of Non flbrations Sobolev Spaces of Fractionary Order. Math. ApI. Comp. (1987).

[9] MEDEIROS L.A., MILLA M. Rernarks on a nonlinear model vibrations of string with dammping. 30 Seminario Brasilero deAnalise LN CC Rl (1989).

[10] ONO K. Global existence, Decay and Blowup of Solutions for Sorne Mildly Degenerate Nonlinear KirchhoffString. J. Diff. Eq. 137 (1997).

[11] PERLA G. On classical solutions of a quasilinear hyperbolic equations. Nonlinear Analysis. Vol. 3(1979).

[12] POHOZAEV 5. The Kirchhoff quasilinear hyperbolic equation. Differential Equations Vol. 21. (1985).

[13] POHOZAEV 5. On a class of quasilinear hyperbolic equation. Math. Sbornik, Vol. 96 (1975).

[14] RIVERA P. On local strong solutions of a nonlinear partial differential equation. AppI. Analysis Vol. 10. (1980).

[15] SIMON J. Cornpact Sets in the Space L $(O, T ; B)$. Universite Pierre et Marie Curie. Laboratoire d' Analyse Numerique (1985).

[16] YAMADA Y. Sorne Nonlinear Degenerate Wave Equations. Non Linear Analysis, 10 (11) (1987).

[17] ZEIDLER E. Non Linear Functional Analysis. Part 1l-B. (1990). 